\title{
After the initial COVID-19 surge: a phased radiology departmental re-opening plan
}

\author{
Victor J. Seghers ${ }^{1,2}$ (D) Nilesh K. Desai ${ }^{1,2} \cdot$ Prakash M. Masand $^{1,2} \cdot$ Sadia Nasir $^{1} \cdot$ Traci L. Foster $^{1} \cdot$ Dennis A. Indiero $^{1}$. \\ Trent D. Johnson ${ }^{1}$ - Thierry A. G. M. Huisman ${ }^{1,2}$
}

Received: 22 May 2020 / Revised: 5 July 2020 / Accepted: 4 August 2020 / Published online: 22 August 2020

(C) Springer-Verlag GmbH Germany, part of Springer Nature 2020

The global coronavirus disease 2019 (COVID-19) pandemic has had a rapid and unprecedented impact upon global health and health care operations [1-3]. In the initial phase of the outbreak, a significant ramping down of nonessential medical care was necessary to prepare hospitals for the forecasted surge in COVID-19 patients. Simultaneously, the spread of the severe acute respiratory syndrome coronavirus 2 (SARS-CoV-2), which causes COVID-19, had to be contained and slowed ("flattening of the curve"). Social distancing, stay home/work safe orders, protective measures for vulnerable individuals (e.g., immunocompromised patients), masking protocols, visitation policies, testing and many more measures resulted in an accelerated but necessary ramping down of elective hospital services [4-9]. All medical specialties, including radiology, were affected. In the last months, many radiology departments experienced a $50-70 \%$ decrease in imaging studies [10]. In addition to its impact on mortality and human suffering, COVID-19's economic impact upon hospital operations has been significant. But what happens when the initial critical phase of the COVID-19 outbreak has passed? Hospital operations must be ramped up again to care for patients whose treatments have been postponed. Unfortunately, a SARS-CoV-2 vaccine is not expected in the near future and an effective treatment is not yet available [11, 12]. Social distancing and masking policies are expected to persist to contain

Victor J. Seghers

vjsegher@texaschildrens.org

1 Edward B. Singleton Department of Radiology, Texas Children's Hospital, 6701 Fannin St., Suite 470, Houston, TX 77030, USA

2 Department of Radiology, Baylor College of Medicine, Houston, TX, USA and slow repeat surges of SARS-CoV-2 infections. Consequently, we must break from the past and accept a new normal in our health care approach. The reopening of a safe radiology service requires a carefully designed plan with creative solutions and lays the foundation for an updated imaging department that incorporates many of the changes implemented during the pandemic. In this manuscript, we present insights for how to design and execute a phased reopening of a radiology department after the pandemic outbreak becomes contained.

\section{Leadership, organization and communication}

A successful plan should follow a concept of Five Cs, which refers to communication, coordination, collaboration, creativity and concentration of resources. Each plan must have well-defined short-, medium- and long-term goals; collect objective metrics for success; and be summarized in a living document that is available to all key players and is updated and adapted in a very dynamic way for unexpected developments. In addition, rules of engagement should be established. Examples of these rules include (a) maintain compliance with local, state and federal guidelines; (b) ensure safety of patients, families and workforce; (c) maximize utilization of technology and automation; (d) optimize throughput and financial performance; and (e) understand and match changing customer preferences with service offerings.

A plan cannot be designed or implemented without a clear leadership and reporting structure. The chief executive officer and president of Texas Children's Hospital, Mark A. Wallace, has defined 10 leadership maxims. One of the most important is, "Leadership always influences or determines outcomes not some of the time, but all of the time." In addition, to optimize the Five Cs we have chosen to follow the "team of teams" concept described by Gen. Stanley McChrystal et al. [13]. The team of teams approach emphasizes a shared 
consciousness and strong lateral connectivity between teams in which people can see the big picture and the interdependent nature of their work. This improved understanding of the problems is crucial to enable empowered execution, in which leaders encourage and nurture decision-making at all team levels.

In our team of teams approach, the Department of Radiology had multiple teams working simultaneously on various goals. One of our first teams was a small group of radiology leaders tasked at the onset of our local COVID-19 crisis to work over a weekend to brainstorm ideas and plan how to ramp up operations. These ideas quickly evolved and were subsequently enshrined in a shared working document where all action items, goals, metrics of success, and timelines were recorded and color-coded to visually demonstrate status and progress (Fig. 1). Progress and obstacles of the various teams are discussed in a daily virtual Microsoft Teams conference (Microsoft Corp., Redmond, WA) where team leaders work on this document and are given daily insight into hospital, city and state status, operations and goals. This team is led by the hospital executive in charge for the Department of Radiology and includes representatives of the various radiology clinical subspecialties (neuroradiology, body radiology and interventional radiology), administrative directors of all hospital campuses, and the community service chief and radiologist-in-chief. While the radiologist-in-chief also participates in daily meetings with other clinical service chiefs and executive leadership for the hospital, the radiologist-in-chief is an integral member of the systemwide "Phased Recovery and Redesign Team" as well, which includes team captains for infection control, surgery, anesthesia, emergency and urgent care centers, radiology, pathology, ambulatory medicine, specialty care centers, e-health, revenue cycle and billing, and marketing and public relations. A human resources representative, legal and risk compliance officer, and a member of the supply chain are consulted as needed.

During these daily meetings, high-level issues regarding COVID-19 testing, current COVID-19-positive patient census, personal protective equipment (PPE) availability, travel guidelines, workforce utilization guidelines, etc., are discussed. In the same meeting, the team captain for radiology presents the relevant details of the radiology reopening progress, which allows for an orchestrated and aligned systemwide reopening of clinical services. The team of teams approach is

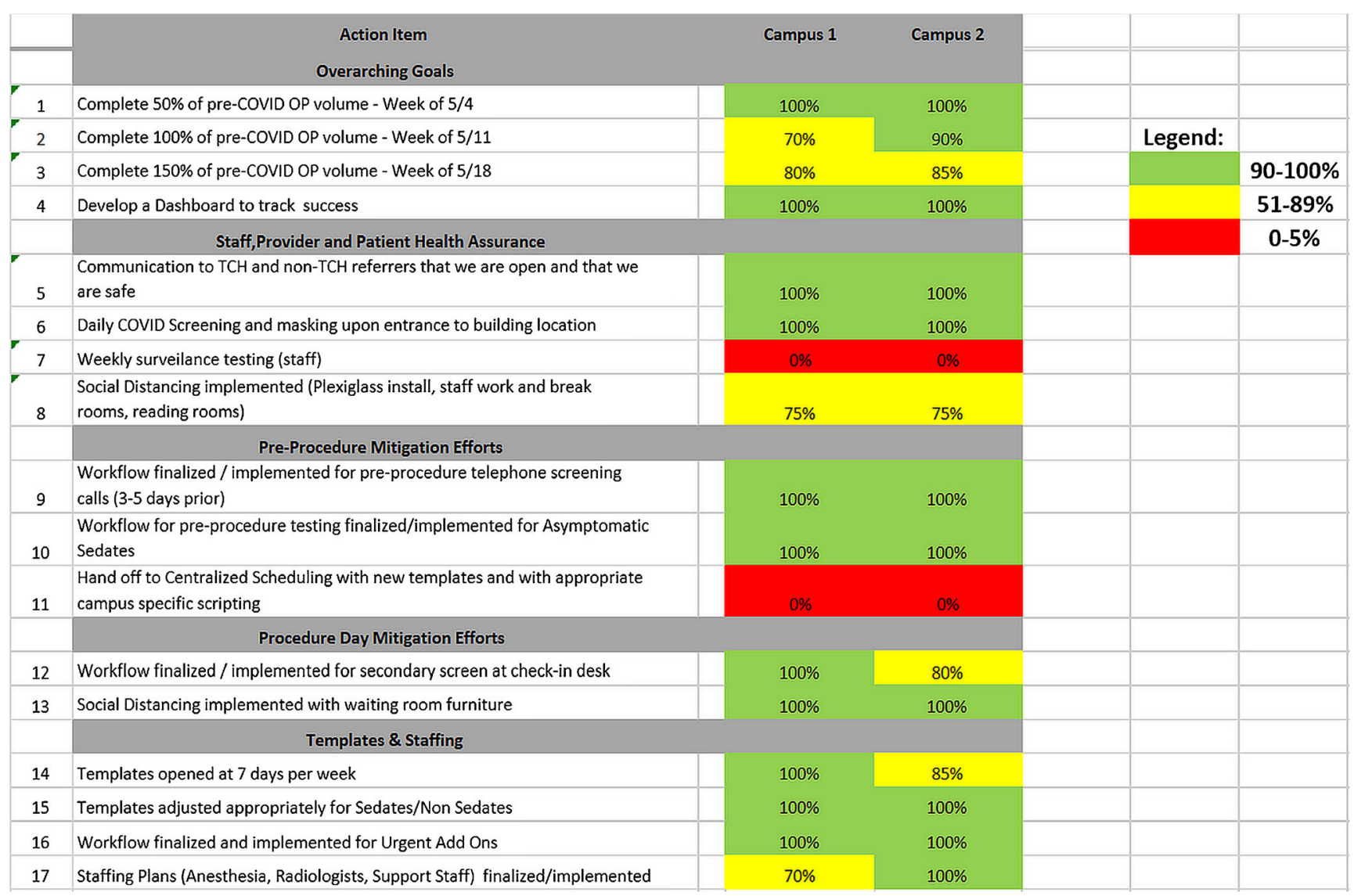

Fig. 1 Sample working document that includes all radiology action items, goals, metrics of success, and timelines. This document should be accessible to all members of the teams, reviewed and updated daily.
Color-coding the various action items and metrics promotes visual demonstration of status and focuses attention on problem areas 
key to ensuring continued alignment among the hospital executives, administrators, and clinical leaders. Although a core group of administrative and clinical radiology leaders was involved in the majority of the teams to provide continuity, particularly as we pivoted from ramping down operations to recovery, team membership was otherwise fluid to better reflect evolving conditions and priorities. To increase buy-in and engagement with the reopening plan as well as communication of information gleaned via roles in overseeing all radiology teams and collaboration with senior executive leadership, the radiologist-in-chief assembles daily progress reports that are communicated to the radiology faculty, staff and administrators.

\section{Setting the goals}

The first step of a successful reopening plan is to set realistic goals and policies, with timing dependent on each institution's local conditions. Planning for a phased reopening is difficult and takes time, and our initial discussion regarding ramping up operations occurred 4 weeks prior to execution. We aimed for a phased reopening with 50\% pre-COVID imaging volume for the first week, followed by $100 \%$ the second week and $150 \%$ the third week. At our institution, the Department of Radiology was one of the first clinical departments to reopen. The impetus for the $150 \%$ imaging volume goal was to ensure that the backlog of unscheduled new imaging orders and the studies that were postponed or cancelled during the initial phase of the COVID-19 outbreak were serviced prior to the expected generation of new imaging orders upon reopening of the ambulatory clinics. Our imaging department did not wish to be a limiting factor for future patient visits and health care management, our clinical partners, and their reopening plans. The $150 \%$ volume targets were achievable through offering new expanded imaging slots during the evenings and on weekends, discussed in greater detail in the "Expanded imaging scheduling grid" section. Scheduling templates and staffing models for the $150 \%$ volume target are temporary and are expected to revert to the $100 \%$ volume target upon servicing the imaging backlog. These goals were carefully aligned with the phased opening of the various clinics within the organization and communicated to hospital providers and private practice referrers. Multiple lines of often duplicative communication is required, whether through global emails, medical staff meetings, administrative leadership forums, websites, departmental faculty meetings, or one-on-one communication. Physician liaisons in departments that are frequent referrers to radiology (otolaryngology, neurosurgery, neurology, endocrinology, gastroenterology, cardiology, urology, orthopedics, and hematology/oncology) were also identified and contacted to accelerate the rescheduling of patients and to communicate that radiology is available to service new outpatient imaging orders. Next to the organizational challenges of flexing up a radiologic service, any plan must address and mitigate fears of the patients and staff that it is safe to come to the hospital. In the following paragraphs we summarize our approach for a systemwide reopening.

\section{Staff health assurance}

It is our responsibility to offer a healthy and, most important, safe SARS-CoV-2 work environment for all our employees. The actions that can slow the COVID-19 outbreak must continue. To effectively diminish employees' fear of getting infected, the organization must aggressively invest in safe workflows. Our policy includes screening of all employees entering the hospital, using a smartphone app via QR code, no-touch thermal imaging cameras for detecting elevated skin temperatures, mandatory masking for everyone, and social distancing markers on the floors throughout the hospital and within the elevators. Departmental break rooms also require social distancing. Signage limiting number of employees in the break rooms is advised as well as the wide availability of disinfectant wipes to clean refrigerators, microwaves, and coffee machines after each use. In addition, a hospital-wide initiative included removing all self-service areas within the restaurants and only offering prepackaged foods. Moreover, wide availability of PPE should be guaranteed and employees that might be exposed to aerosolizing procedures or COVID19 patients should be fitted for an N-95 mask in advance [14]. Finally, wide access of the staff and faculty to rapid testing after unprotected exposure to a positive patient or expression of symptoms including both direct virus detection and antibody testing is essential. Collaboration with the infection control team regarding PPE utilization, definition of aerosolgenerating procedures, and testing policies is important to ensure that departmental decisions are in compliance with the institution and evidence-driven. Whenever possible, surveillance testing on a regular basis for the staff most exposed should be offered.

\section{Patient health evaluation and assurance}

Much planning and effort are required before imaging procedures for multiple categories of patients including hospital and remote-site outpatients, inpatients, urgent care and emergency center patients. Depending on the local settings of each radiology department, various combinations of policies might be implemented and are summarized in Table 1.

At our institution, prior to their scheduled imaging appointment patients receive messaging via phone, text or mail describing what to expect upon arrival at the hospital or imaging center and reassurance that extensive 
Table 1 Staff and patient health evaluation and assurance

1. Pre-imaging phone call explaining to patients what to expect on arrival and reassurance that extensive measurements are being followed to limit exposure

2. Pre-visit screening on the phone $24-48 \mathrm{~h}$ prior to the date of imaging

3. On-site hospital entrance QR code-assisted screening with secondary screening at welcome desks

4. Temperature measurements, masking, etc.

5. Parking-lot check-in

6. Secondary screening at radiology welcome desk documented with extra "dot" on name tag

7. Plexiglas shielding of all welcome desks

8. Uniform masking of all patients and employees

9. Single parent visitation

10. Pre-visit SARS-CoV-2 testing for all sedated/intubated patients, drive-through testing $24-48 \mathrm{~h}$ prior to date of imaging

11. PPE for all aerosolizing imaging and interventional procedures

12. N-95 mask fitting for at-risk staff and faculty

13. Daily metrics including \% no-shows

14. Extended hours allowing for more time spacing between imaging studies

15. Home workstations for all faculty

16. Identification of faculty at risk to primarily support clinical services from home ( $>65$ years old, immunocompromised, etc.)

17. Daily updates for faculty and staff

18. Consider separate IP and OP scanner utilization/scheduling

19. Communication strategy to alert hospital providers and private practices that radiology is open and safe

20. Identification of clinical liaisons outside radiology to better triage and accelerate patient rescheduling

21. Value of a phased reopening plan, 50-100-150-100\%

22. Cancellation of academic days/administrative days for faculty in preparation of reopening and surge of postponed studies

23. Academic travel restrictions until the end of the year in order to upgrade the clinical task force availability

24. Work-volume-linked dynamic flexing down and flexing up of staff

$h$ hours, $I P$ inpatient, $O P$ outpatient, $P P E$ personal protective equipment, $Q R$ quick response, $S A R S-C o V-2$ severe acute respiratory syndrome coronavirus 2

procedures have been enacted to limit exposure and assure their safety. Because hospital and departmental safety procedures are expected to evolve over time, it is important to provide a link to a website in the messaging to ensure the patients always have access to the most upto-date information [15]. Additional pre-visit screening for symptoms concerning for COVID-19 at least $48 \mathrm{~h}$ prior to the date of imaging should also be performed. For imaging exams not requiring sedation, these phone calls can be made by modality technologists and documented in the electronic medical record. Patients who fail screening can have their imaging appointment postponed for 2 weeks or be referred for COVID-19 testing. At our institution, only outpatients undergoing procedures requiring sedation are automatically tested for SARS-CoV-2. Department-approved scripting can enable streamlined and consistent communication, and ensure that crucial information is conveyed appropriately. In addition to further reassuring patients of the numerous safety procedures that exist, these calls also serve to decrease incidence of noshow appointments. Current radiology staffing is likely significantly reduced and increased staffing is needed to accommodate any reopening plan, so ensuring that patients present for their appointments is crucial.

To support social distancing, only one family member should be allowed with the patient on the day of the imaging appointment. A patient screening process with mandatory masking at designated entrances of the hospital should be performed, similar to what has been described for employees, with additional secondary screening at radiology check-in locations. A virtual screening and check-in process performed via smartphone app and $\mathrm{QR}$ code and linked to the electronic medical record can ensure that wait times are kept to a minimum. This process could also be utilized to instruct patients to call the radiology front desk upon arrival at the parking lot of the hospital. Depending on the number of people in the waiting room, radiology personnel would request they remain in their car until notified to appear for their imaging exam. Processes to ensure short wait times for families can be created to support patient satisfaction metrics. Escalation procedures should be established for when patients or families refuse to wear masks or have questions regarding safety. Welcome desks in radiology reception areas should have plexiglass shielding installed to limit potential exposure between patients and staff, and waiting rooms should be redesigned to limit the number of people present at any one time. Removing existing seating and converting seating from cloth- to vinyl-covered seats to make cleaning easier can be quick and cost-effective methods.

All outpatients undergoing interventional radiology procedures or imaging exams requiring sedation are screened at least $48 \mathrm{~h}$ in advance by radiology nursing staff, documented in the electronic medical record, and scheduled for COVID-19 testing, preferably via drivethrough testing locations at all radiology locations (Fig. 2). The ability to perform rapid testing with results available in a few hours at all imaging locations allows screening and testing to be performed as close to the imaging appointment as possible and is particularly helpful to enable a single visit for out-of-town patients. To streamline workflows and decrease need to contact ordering physicians, radiologists place orders for testing. All symptomatic patients who fail radiology screening or test positive for COVID-19 report to a dedicated clinic staffed by anesthesia providers who assess the patients and make treatment and disposition recommendations. 


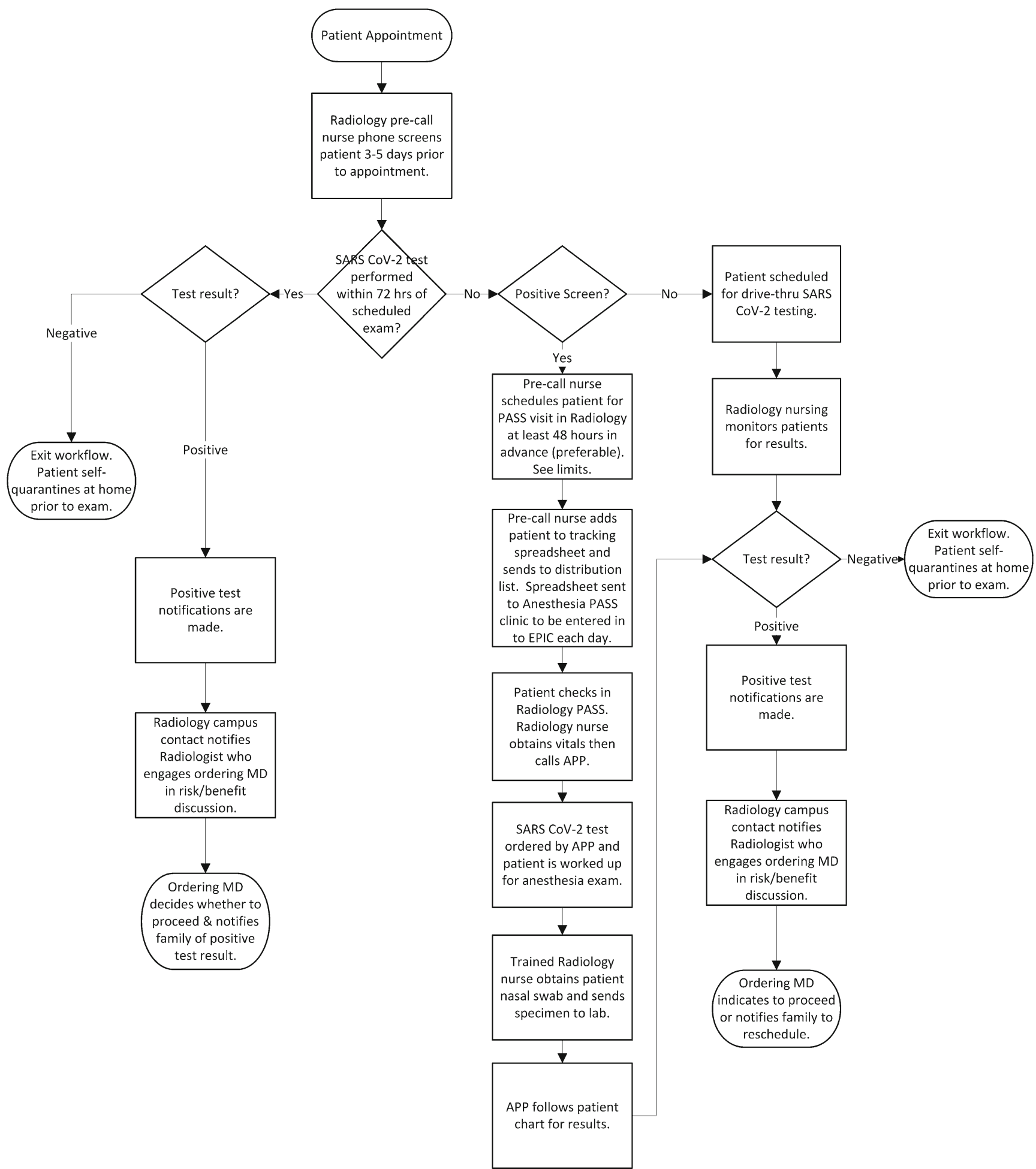

Fig. 2 Outpatient screening and testing workflows in preparation for procedures requiring sedation. $A P P$ advanced practice provider, $M D$ medical doctor, $P A S S$ pediatric anesthesia screening service (a dedicated clinic staffed by anesthesiologists and nurse practitioners who

Hospital inpatients should be tested for COVID-19 upon admission and again for new or progressive symptoms. Patients presenting to the emergency department or screen patients with conditions that might increase risk during anesthesia), SARS CoV-2 severe acute respiratory syndrome coronavirus 2

inpatients who require emergent imaging are treated as a person under investigation (PUI) requiring full PPE on the part of radiology staff. 


\section{Expanded imaging scheduling grid}

To achieve pre-COVID-19 imaging volumes, work through the backlog of cases rescheduled in preparation for the expected surge of patients, and support social distancing measures to prevent patient clustering, multiple changes can be considered including shorter imaging protocols, reduced research in favor of clinical exam volume, and scheduling COVID-19-positive patients separately from negative patients. We have found that offering expanded scheduling slots during weekday evening hours and on weekends is a quick and effective response. This is difficult with existing staffing models, even more so if the hospital is reluctant to bring back flexed down or furloughed staff as a result of fiscal and operational uncertainty. Constant attention to percentage of filled imaging slots and no-shows allows for dynamic flexing up or down of staff relative to work volume, and for discovering trends and making adjustments in templates and workflows. This process is challenging because not all modalities or physical locations are equally staffed. Identifying the hours of operation for each modality, weekdays and weekends, as well as availability for sedated and non-sedated slots (e.g., MRI and interventional radiology) is necessary. Ensuring that sufficient gaps between imaging slots exist to prevent patient clustering is required. Many interdependent variables need to be considered in creating these slots, and communication is crucial. For example, a particular modality might have sufficient technologists and radiologists but lack nursing or front desk support staff. Awareness of operations in other areas of the hospital is important because not all clinics will re-open at the same speed, particularly the lower-revenue-generating departments. This creates the opportunity to utilize non-radiology departmental staff throughout the hospital who might otherwise be furloughed but can be flexed up earlier and more productively.

Expanded access for sedation slots is likely to be challenging because anesthesia partners will also be utilized aggressively in surgical re-opening plans. However, creative thinking among team members can overcome some challenges of scarcity. For example, if in order to better manage patient throughput certain units or rooms will be temporarily shut down, some efficiencies could occur such as creation of dedicated sedation and non-sedation MR units or operations to better separate inpatient from outpatient volumes. As the new scheduling grids are built and backfilled by patients rescheduled in preparation for the surge, it might be easier initially to bypass centralized scheduling departments whose staff are flexed down in favor of manual scheduling within radiology, whose leaders are familiar with the newly created expanded imaging schedules. However, as radiology operations increase and staff members are needed to handle larger imaging volumes, a transition back to centralized scheduling might be beneficial.

\section{Radiologist safety and staffing plan}

During the initial phase of the COVID-19 outbreak, faculty and staff who were most at risk (e.g., $>65$ years of age, immunocompromised) were identified and prioritized to provide clinical services from home. The remaining faculty were placed in non-overlapping teams either working from home or spaced apart in reading rooms to promote social distancing. For institutions interested in developing or broadening home capability for the picture archiving and communication system (PACS), a practical guide including technical specifications has recently been published [16]. Our trainees were sent home, and research efforts were significantly curtailed. Clinical conferences, administrative meetings and individual case discussions with trainees or colleagues are held virtually, using a mixture of Zoom (Zoom Technologies, San Jose, CA), Webex (Cisco Systems, San Jose, CA) and Microsoft Teams. For large groups, it has been helpful to use web cameras and chat features of the video conferencing platforms to improve engagement and efficiency of discussion. Additionally, all at-risk faculty and staff were fitted for N-95 masks, and we developed policies ensuring PPE usage for all aerosolizing imaging and interventional procedures. With low clinical volumes, many academic hospitals have encouraged radiologists to take vacation or have temporarily ceased financially supporting meeting and academic time in order to upgrade the clinical taskforce availability in expectation of reopening plans and a surge of postponed examinations. At our institution, physician and executive salaries have been temporarily reduced by $10-20 \%$, and radiologists were instructed to use up to $50 \%$ of their annual vacation by June 30, 2020, and only schedule 1 week of vacation over the summer to ensure clinical coverage in light of expanded imaging templates afterhours, on weekdays and on weekends.

In designing a reopening plan, on-site radiologist staffing needs to increase in accordance with increased clinical volumes. Radiologists provide a necessary support to referring clinicians beyond their ability to provide image interpretation. Leadership within the department and greater clinical integration are needed to ensure that radiology does not simply become a commodity in these financially challenged times. Nevertheless, in the absence of a SARS-CoV-2 vaccine, social distancing practices will need to continue, which will affect rotational workflows and reading room occupancy rules. Guidelines are needed to ensure that most radiologists continue to work in the hospital while leveraging the benefits of home PACS to equitably address health concerns, improve commuting times, increase morale and mitigate issues of burnout, and extend coverage in the evenings and on weekends without the need to be on-site [17]. 


\section{Daily assessment/metrics of success}

A radiology department ramp-up plan will involve many people, teams and interdependent variables, and it is important to perform daily assessments and determine metrics of success. Creation of virtual dashboards enables rapid assessment and dissemination of information and trends. Daily communication of the number of COVID-19 tests performed on patients and staff, positive tests, quarantined staff, testing locations, and amount of available PPE is important to assure staff that they remain safe despite efforts to re-open operations. Once expanded scheduling templates have been created, prospective analysis of how many slots have been filled for the next day and week will help justify increased labor costs of staffing. Retrospective assessment of the daily imaging volumes and no-show rate will further verify that staffing is in alignment with imaging volumes and that the scheduling and communication plan is working. As many non-urgent exams are rescheduled to accommodate the expected surge of COVID-19-positive inpatients, evaluation over time of the backlog of exams to be scheduled will determine whether the new expanded templates are sufficient or need to be adjusted. This analysis of orders to be scheduled is also an important metric of the future expected volume for the radiology department and can be used to assess how rapidly clinical departments who frequently refer to radiology are reopening operations. As mentioned, anesthesia support for expanded sedation slots might be challenging, so utilization of pre-determined metric thresholds such as at least $70 \%$ utilization of sedation slots will help justify additional requests for service. Finally, financial analysis of current operations relative to budget is necessary to judge performance of the rampup plan. Through daily, weekly and monthly assessment, trends will be discovered that can be used to create positive momentum for staff, faculty and administration and further help achieve buy-in for the plan.

\section{From crisis to opportunity}

"A crisis is a productive state. You simply have to get rid of its aftertaste of catastrophe."

- Max Frisch, Swiss playwright and novelist (19111991)

The coronavirus pandemic has been a trial by fire in crisis management. Radiology leaders have had to make difficult, stressful and quick decisions while projecting a sense of calm and confidence. As radiology departments begin recovery operations, some will attempt to restore familiar routines, processes and workflows. However, to ignore the opportunity to redesign a post-COVID-19 radiology service is a mistake. Prior to the pandemic, all our departments had vexing issues to a certain degree, ranging from small to large, and insignificant to critical. The overwhelming need to expand operations to reclaim volume and finances while maintaining access and safety for patients and staff means that there has never been a better opportunity to create positive and lasting change [18]. For guidance to what changes should be considered, a focus on our patients and attention to trends already occurring before the pandemic is helpful (Table 2). This can include patient-directed online scheduling and expanded access to imaging, offering same-day service with hours and locations adapted to the patient and family lifestyle; improved use of virtual dashboards to more easily track various metrics including MR efficiency, sedation utilization, and length of patient stay in the imaging department; and investment in

Table 2 Radiology: the opportunity to re-design operations post COVID-19

1. Flexible opening hours adapted to patient and family lifestyles

2. Bringing imaging to the patient: imaging sites within shopping centers, mobile ultrasound units close to schools or sports centers

3. Online scheduling using decision-tree algorithms to ensure patients can schedule themselves at the right place, the right time and on the right camera

4. Combined imaging with other diagnostic services in standalone diagnostic centers, e.g., pathology or phlebotomy services

5. Combined imaging with other after-hours services such as physical therapy for children with musculoskeletal (MSK) injuries

6. Continued development of subspecialty pediatric radiology expertise (neurology, interventional, nuclear, cardiac, MSK, fetal radiology) and offering 24/7 teleradiology services to small and mid-size adult hospitals

7. Extended telemedicine to interventional radiology (IR) services, e.g., vascular anomalies clinic, individual IR clinic appointments

8. Developed at-home work guidelines for radiologists to leverage benefits of home PACS

9. Expanded walk-in services, e.g., same-day imaging + clinic visit service

10. Redesigned radiology research program to better develop academic careers and focus on topics that match the expertise of the institution

11. Improved departmental website and social media strategy to better communicate with patients

12. Improved MR efficiency, e.g., standardization and shorter imaging protocols, separation of inpatients \& outpatients, sedated and non-sedated patients, alternative methods to sedation such as mock MRI scanner, child life specialists, therapy animals

13. Greater use of virtual dashboards and patient imaging/care coordinators to improve patient throughput and increase patient satisfaction

14. Investment in leadership development, diversity and inclusion, physician wellness

15. Prioritized value over imaging volume through evidence-based clinical decision support

PACS picture archiving and communication systems 
telemedicine and teleradiology platforms to better enable pediatric radiology subspecialty expertise to come to the patient, no matter their location. While a primary focus for any initial departmental re-opening plan will be to reclaim imaging volumes, national health care trends prioritize value of imaging services along with utilization of evidence-based clinical decision support $[19,20]$. The Black Lives Matter movement has renewed focus on how implicit physician bias as well as education, poverty and other social determinants of health contribute to health care disparities among racial groups, most acutely the coronavirus death rate and access to SARS-CoV-2 testing and treatment. Greater awareness of the negative impact of implicit bias within radiology departments as well as recruitment and mentorship programs to prioritize diversity and inclusion can ensure that faculty and staff better reflect the communities in which they serve. Creative solutions to these trends in light of the global pandemic will obviously depend on an individual department's culture, needs and local environment.

\section{Conclusion}

The current global health conditions required a rapid response to ramp down operations to ensure safety to patients and staff and ensure bed capacity for the expected surge of COVID-19positive patients. Now that the initial surge is passing in many parts of North America, radiology departments need to pivot and just as rapidly implement re-opening plans in alignment with loosening of government stay-at-home, work-safe orders. Institutional culture is of paramount importance to be able to to lead change.

At the time of initial manuscript submission, North America appeared to be weathering the current storm, but as of the last week of June, $22 \%$ of all people in the Houston metropolitan area tested for SARS-CoV-2 were positive and our pediatric institution had agreed to accept a limited number of adult SARS-CoV-2-positive and negative patients to support our community [21]. We cannot become complacent and need to retain and further evolve our response. Our institution's investment in testing capabilities and supply chain processes enables us to continue to provide a safe work environment for our patients and staff despite this increase in SARSCoV-2-positive patients, and we continue to provide emergent and elective imaging services in accordance with local and state mandated orders. However, the implication of this increase in positive tested individuals on our recovery plan is still unknown. As the global COVID-19 pandemic continues to evolve, we hope to provide an update to our experience and response in this forum. The magnitude of the challenges suggests that attempts to return to familiar routines and workflows is misguided, and instead provides an opportunity for reinvention of radiology departmental operations.

\section{Compliance with ethical standards}

Conflicts of interest None

\section{References}

1. Li W, Cui H, Li K et al (2020) Chest computed tomography in children with COVID-19 respiratory infection. Pediatr Radiol 50: 796-799

2. Rubin GD, Ryerson CJ, Haramati LB et al (2020) The role of chest imaging in patient management during the COVID-19 pandemic: a multinational consensus statement from the Fleischner Society. Chest 158:106-116

3. Zhu N, Zhang D, Wang $\mathrm{W}$ et al (2020) A novel coronavirus from patients with pneumonia in China, 2019. N Engl J Med 382:727733

4. Czernin J, Fanti S, Meyer PT et al (2020) Nuclear medicine operations in the times of COVID-19: strategies, precautions, and experiences. J Nucl Med 61:626-629

5. Mossa-Basha M, Meltzer CC, Kim DC et al (2020) Radiology department preparedness for COVID-19: radiology scientific expert panel. Radiology. https://doi.org/10.1148/radiol.2020200988

6. Pandey AS, Ringer AJ, Rai A et al (2020) Letter: considerations for performing emergent neurointerventional procedures in a COVID19 environment. Neurosurgery. https://doi.org/10.1093/neuros/ nyaa 173

7. Qu J, Yang W, Yang Y et al (2020) Infection control for CT equipment and radiographers' personal protection during the coronavirus disease (COVID-19) outbreak in China. AJR Am J Roentgenol. https://doi.org/10.2214/AJR.20.23112

8. Revel M-P, Parkar AP, Prosch H et al (2020) COVID-19 patients and the radiology department - advice from the European Society of Radiology (ESR) and the European Society of Thoracic Imaging (ESTI). Eur Radiol. https://doi.org/10.1007/s00330-020-06865-y

9. Zixing H, Shuang Z, Zhenlin L et al (2020) The battle against coronavirus disease 2019 (COVID-19): emergency management and infection control in a radiology department. J Am Coll Radiol 17:710-716

10. Cavallo JJ, Forman HP (2020) The economic impact of the COVID-19 pandemic on radiology practices. Radiology. https:// doi.org/10.1148/radiol.2020201495

11. Amanat F, Krammer F (2020) SARS-CoV-2 vaccines: status report. Immunity 52:583-589

12. Lu S (2020) Timely development of vaccines against SARS-CoV2. Emerg Microbes Infect 9:542-544

13. McChrystal S, Collins T, Silverman D, Fussell C (2015) Team of teams: new rules of engagement for a complex world. Penguin Books, New York

14. Society of Interventional Radiology (2020) Aerosol generating procedures performed by interventional radiology clinical notification from the Society of Interventional Radiology. SIR COVID-19 resources for IR website. https://www.sirweb.org/practice-resources/ covid-19-resources/covid-19-clinical-notification-3-26-20/. Accessed 15 July 2020

15. Texas Children's Hospital (2020) Always here for you. TCH COVID-19 updates webpage. texaschildrenscoronavirus.org. Accessed 15 July 2020

16. Sammer M, Sher A, Huisman T, Seghers V (2020) Response to the COVID-19 pandemic: practical guide to rapidly deploying home workstations to guarantee radiology services in times of quarantine, social distancing or stay at home orders. AJR Am J Roentgenol. https://doi.org/10.2214/AJR.20.23297 
17. Yamashiro T, Kumamaru K, Kido A et al (2020) Work-style reform and use of information and communication technology among diagnostic radiologists in Japan: results of the 2018 JRS/JCR joint survey. Jpn J Radiol 38:636-642

18. Walker S (2020) Getting the restart right: how to lead when nobody has a map. Wall Street Journal. https://www.wsj.com/articles/ getting-the-restart-right-how-to-lead-when-nobody-has-a-map11587787201. Accessed 15 July 2020

19. Norbash A, Bluth E, Lee CI et al (2014) Radiologist manpower considerations and imaging 3.0: effort planning for value-based imaging. J Am Coll Radiol 11:953-958
20. Sarwar A, Boland G, Monks A, Kruskal JB (2015) Metrics for radiologists in the era of value-based health care delivery. Radiographics 35:866-876

21. Texas Medical Center (2020) Coronavirus (COVID-19) updates. TMC website. https://www.tmc.edu/coronavirus-updates/. Accessed 15 July 2020

Publisher's note Springer Nature remains neutral with regard to jurisdictional claims in published maps and institutional affiliations. 